\title{
Análise epidemiológica dos pacientes acometidos por Degeneração Macular Relacionada à Idade, atendidos no Setor de Retina da Faculdade de Medicina do ABC
}

\author{
Epidemiological Analysis of patients diagnosed with Age-Related Macular \\ Degeneration in Retina's Sector of Faculdade de Medicina do ABC
}

Dérica Serra', Rodrigo Angelucci', Débora Sugano', Paulo Ricardo Sampaio', José Ricardo Rehder'

\begin{abstract}
Resumo
Objetivo: 0 objetivo do presente estudo foi analisar o perfil epidemiológico dos pacientes acometidos por Degeneração Macular Relacionada à Idade (DMRI), atendidos no Setor de Retina e Vítreo do Instituto de Olhos da Faculdade de Medicina do ABC - SP (FMABC). Casuística e Métodos: Realizouse um estudo transversal que avaliou 1179 prontuários de pacientes atendidos no Setor de Retina do Instituto de Olhos da FMABC, no período de janeiro de 2003 a janeiro de 2004. Os pacientes que apresentaram diagnóstico de DMRI foram analisados quanto ao sexo, idade, acuidade visual e forma da DMRI. Resultados: Dos 1179 prontuários avaliados, 92 (7,8\%) pacientes apresentaram diagnóstico de DMRI. Em relação à forma clínica da DMRI observou-se 106 (64,63\%) olhos com a forma atrófica, e 58 (35,37\%) olhos com aspectos exsudativos da doença, sendo 32 (59\%) com sinais cicatriciais e 26 (41\%) com neovascularização sub-retiniana. Conclusões: A DMRI é mais freqüente em pacientes acima dos 75 anos.
\end{abstract}

Unitermos: Maculopatia relacionada à idade; visão subnormal; epidemiologia; cegueira; qualidade de vida.

Abstract

Objective: The objective of this paper was to evaluate the epidemiology of the patients diagnosed with Age-Related Macular Degeneration (AMD) in Retina's Sector of Faculdade de Medicina do ABC - SP (FMABC). Subjects and Methods: A cross-sectional study was accomplished to evaluate 1179 patients records at FMABC Eye Institute, Retina sector, from January 2003 until January 2004. Patients with AMD were analyzed about sex, age, visual acuity and AMD form. Results: Ninety-two patients (7.8\%) presented AMD among 1179 evaluated records. It was noted 106 (64.63\%) cases of atrophic form, concerning AMD clinical form and $58(35.37 \%)$ cases with exsudative aspect of the disease. Thirty two of them (59\%) presented scarring and 26 (41\%) presented signs of sub retinal neovascularization. Conclusion: AMD showed to be more frequent in patients after 75 years old.

Keywords: Macular Degeneration; vision, low; epidemiology; blindness; quality of life.

Recebido: 28/02/2007

Revisado: $22 / 09 / 2007$

Aprovado: $18 / 03 / 2008$

Trabalho realizado no Instituto de Olhos da Faculdade de Medicina do ABC (FMABC), Santo André (SP), Brasi

Disciplina de Oftalmologia da FMABC

Endereço para correspondência: Rodrigo Interlandi Angelucci, Alameda Joaquim Eugênio de Lima, 1742, CEP $01425-011$ - São Paulo (SP)

E-mail: rodrigoangelucci@yahoo.com.br 


\section{Introdução}

A Degeneração Macular Relacionada à Idade (DMRI) é considerada atualmente a maior causa de cegueira legal nos países industrializados ${ }^{1-4}$.

Estudos revelam que a prevalência da DMRI pode ocorrer em $1,2 \%$ dos pacientes com menos de 65 anos de idade, $7 \%$ entre 65 e 74 anos e aproximadamente em $20 \%$ dos pacientes com idade superior aos 75 anos $^{4-5}$

O Macular Photocoagulation Study Group considera como DMRI a presença de drusas associadas a alterações pigmentares na mácula, em indivíduos com idade acima de 50 anos 6

A DMRI pode se apresentar nas formas não exsudativa (seca ou atrófica) e exsudativa (úmida) ${ }^{6}$.

A forma atrófica, a mais freqüentemente encontrada, corresponde a $20 \%$ dos casos com perda visual grave, enquanto a forma exsudativa é responsável por $80 \%$ deles $^{2-4,7}$.

A perda de visão na DMRI seca é causada por uma disfunção metabólica do epitélio pigmentário da retina (EPR) com conseqüente atrofia das células fotoreceptoras. Na maioria dos casos, a baixa de visão é lenta e gradual, com acometimento inicial do campo de visão central e posteriormente perda na qualidade da visão. No entanto, geralmente esta perda não é tão importante quanto à provocada pela DMRI úmida, que pode apresentar sintomas de metamorfopsia, precedendo uma baixa de visão rapidamente progressiva em decorrência da formação de membranas neovasculares subretinianas (MNVSR).

Na DMRI exsudativa, algumas formas de tratamento mostram-se eficazes no controle da evolução da doença, como a terapia fotodinâmica e medicações anti-angiogênicas ${ }^{3,6-7}$

Nos casos em que a visão residual apresenta-se insatisfatória, o paciente deve ser acompanhado conjuntamente em um serviço de visão subnormal (VSN) ${ }^{1,3}$. Até o presente momento, os auxílios ópticos representam uma das poucas alternativas de tratamento para a melhoria da qualidade de vida da maioria desses pacientes, que geralmente apresentam a visão periférica preservada ${ }^{1-2,7}$.

Existem várias definições e classificações para VSN ou baixa de visão. Considera-se como portador de VSN o paciente com acui-

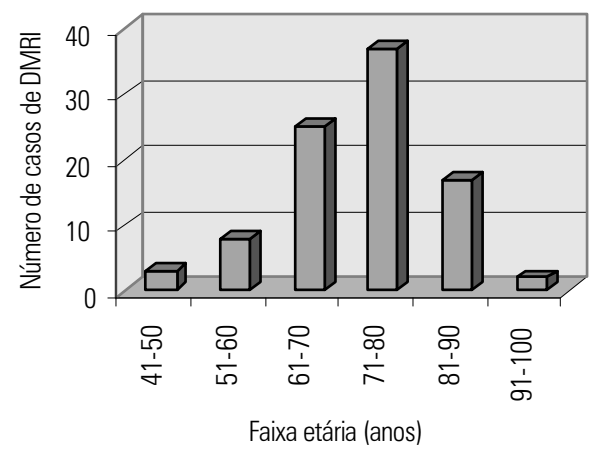

Figura 1 - Distribuição do diagnóstico de DMRI por faixa etária. dade visual inferior ou igual a 20/60 no melhor olho com a melhor correção obtida com meios usuais, como óculos e lentes de contato ou com campo visual menor que $10^{\circ}$, mas que utiliza ou é potencialmente capaz de utilizar a visão para o planejamento ou execução de uma tarefa ${ }^{8-9}$.

Outro conceito que deve ser ressaltado é o de cegueira legal, no qual o paciente é portador de acuidade visual igual ou menor que $20 / 200$, ou campo visual menor ou igual a $10^{\circ} \mathrm{em}$ seu melhor olho, com melhor correção ${ }^{10}$.

O objetivo do presente estudo foi analisar o perfil epidemiológico dos pacientes acometidos por DMRI, atendidos no Setor de Retina e Vítreo do Instituto de Olhos da Faculdade de Medicina do ABC - SP (FMABC), no período de janeiro de 2003 a janeiro de 2004.

\section{Casuística e Métodos}

Foi realizado um estudo transversal que avaliou os prontuários de 1179 pacientes atendidos no Setor de Retina e Vítreo do Instituto de Olhos da FMABC, no período de janeiro de 2003 a janeiro de 2004.

Os pacientes que apresentaram diagnóstico de DMRI foram analisados quanto ao sexo, idade, acuidade visual e forma da doença.

Foram considerados portadores de DMRI os pacientes com drusas associadas a alterações pigmentares na mácula e idade superior a 40 anos.

Foi realizada a análise descritiva de todas as variáveis, as quais foram apresentadas em valores absolutos e relativos.

\section{Resultados}

Dos 1179 prontuários avaliados, 92 (7,8\%) pacientes apresentaram diagnóstico de DMRI.

Em relação ao gênero 40,3\% dos pacientes eram do sexo masculino e $59,7 \%$ do sexo feminino.

Quando avaliada a faixa etária dos pacientes, observou-se uma variação entre 46-92 anos com predomínio dos casos entre 71 - 80 (40,22\%) anos de idade (Figura 1). A idade média encontrada foi de 72,5 anos.

$\mathrm{Na}$ Tabela 1 podem ser observadas as acuidades visuais encontradas.

Tabela 1 - Distribuição numérica e percentual das acuidades visuais

\begin{tabular}{lcc}
\hline Acuidade visual & $\mathrm{N}^{0}$ de olhos & Porcentagem \\
\hline $20 / 30$ a 20/60 & 66 & $35,86 \%$ \\
\hline $20 / 80$ & 09 & $4,90 \%$ \\
\hline $20 / 100$ & 16 & $8,70 \%$ \\
\hline $20 / 125$ & 03 & $1,63 \%$ \\
$20 / 160$ & 09 & $4,90 \%$ \\
\hline $20 / 200$ & 11 & $5,97 \%$ \\
$20 / 400$ & 06 & $3,26 \%$ \\
\hline $20 / 400$ ou pior & 64 & $34,78 \%$ \\
\hline Total & 184 & $100,00 \%$ \\
\hline
\end{tabular}




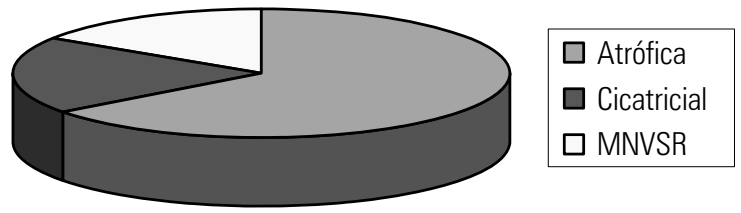

Figura 2 - Proporção das formas clínicas de DMRI.

Dos 184 olhos avaliados, $164(89,13 \%)$ possuíam algum tipo de DMRI e $20(10,87 \%)$ não apresentaram alterações oftalmoscópicas e angiográficas visíveis, sendo considerados normais.

Em relação à forma clínica da DMRI observaram-se 106 (64,63\%) olhos com a forma atrófica, e 58 (35,37\%) olhos com aspectos exsudativos da doença, sendo 32 (59\%) com sinais cicatriciais e 26 (41\%) com alguma forma de atividade de neovascularização subretiniana (MNVSR) (Figura 2).

Dos 92 pacientes com diagnóstico de DMRI, 57 (62\%) eram portadores de VSN. Destes, 21 (36,8\%) se enquadravam na definição de cegueira legal, sendo a DMRI exsudativa responsável por $75 \%$ desses casos.

\section{Discussão}

Dos 1179 prontuários analisados no setor de Retina e Vítreo do Instituto de Olhos da FMABC pôde-se observar uma prevalência de $7,8 \%$ de pacientes com diagnóstico de alguma forma de DMRI, coincidindo com a prevalência encontrada na população geral de países industrializados, que varia entre $5,8 \%$ a $8,8 \% \%^{4-5}$. Até o presente momento não se dispõem de dados referentes à incidência da DMRI.

Em relação ao gênero, o sexo feminino foi mais acometido do que o masculino. Uma possível explicação seria a maior sobrevida das mulheres e o fato destas consultarem o oftalmologista com uma maior freqüência que os homens. Apesar da alta prevalência do sexo feminino na maioria dos estudos, parece não haver nenhuma relação significante entre a DMRI e o sexo ${ }^{5,11}$.

Quando analisados os grupos etários da presente amostra, a maior incidência de indivíduos acometidos encontrou-se na faixa entre 7180 anos, corroborando os estudos da literatura revisada que demonstram aumento da prevalência de DMRI, independente da forma clínica, de acordo com o avanço da idade ${ }^{4-5,12}$. Ressalta-se que a idade média de acometimento de DMRI nesta pesquisa foi de 72,5 anos, coincidindo com os estudos de Owen et al. que encontraram uma média de 75 anos de idade ${ }^{12}$. Vale salientar que neste trabalho foram computados pacientes abaixo de 50 anos de idade porque estes apresentavam sinais associados à degeneração macular.

Quanto à apresentação clínica da DMRI, a forma atrófica foi a mais freqüentemente encontrada, com aproximadamente $65 \%$ dos olhos analisados; e a forma exsudativa, apesar de menos freqüente, mostrou-se responsável por $75 \%$ dos casos de comprometimento visual severo, concordando com os trabalhos estudados ${ }^{2-3,7}$

Em uma análise de 100 pacientes atendidos no setor de VSN da FMABC, Angelucci et al., relataram que 43\% desses pacientes foram encaminhados pelo Setor de Retina e Vítreo, sendo a DMRI responsável pela segunda maior incidência dos casos, seguida da coriorretinite por toxoplasmose ${ }^{13}$. Tal constatação deve alertar que as doenças retinianas, principalmente a DMRI, são responsáveis por grande parcela de indivíduos com baixa visão e cegueira e que esses casos necessitam de acompanhamento em serviços especializados em baixa visão.

O conceito atual em relação à reabilitação visual do paciente idoso com visão subnormal ou baixa visão é que, paralelamente ao tratamento oftalmológico - clínico ou cirúrgico - se realizem as condutas ópticas e reabilitacionais ${ }^{14}$.

Em diversos países do mundo pode-se encontrar serviços de visão subnormal consolidados e bem equipados. No Brasil, nota-se que ainda há carência nesta área ${ }^{15}$.

O Serviço de Visão Subnormal da disciplina de Oftalmologia da FMABC teve sua implantação no segundo semestre de 2001, com o intuito de conduzir o grande contingente de pacientes portadores de baixa de visão da região do Grande $\mathrm{ABC}$ e ensinar aos médicos oftalmologistas integrantes da disciplina como orientar esses pacientes ${ }^{16}$. Atualmente, o setor funciona com uma equipe multidisciplinar que agrega profissionais especializados em educação especial para Braille, terapia ocupacional para orientação e mobilidade, psicologia, psicopedagogia, fisioterapia, atividades da vida diária e oftalmologistas, promovendo, desta forma, um atendimento integral aos pacientes. Assim, a análise dos resultados demonstra claramente que a DMRI pode tornar-se um importante problema de saúde pública no futuro próximo e que existe a necessidade do acompanhamento dos pacientes portadores desta doença por um setor de visão subnormal, com o intuito de proporcionar a eles melhor qualidade de vida.

Concluímos que a DMRI é mais freqüente em pacientes acima dos 75 anos. 


\section{Referências}

01. Ducrey N, Goldschmidt M, Moroszlay S, Moreau D, Schlittler A, Simon F. Follow-up of patients suffering from age-related macular degeneration, supplied with visual aids. Klim Monatsbl Augenheilkd 2000;216(5):278-82.

02. Murphy RP. Age-related macular degeneration. Ophthalmology 1986;93:969-71.

03. Bressler SB. Health maintenance issues of the elderly. Vision: age-related degeneration. Md Med J 1989;38(2):135-7.

04. Soubrane G, Haddad WM, Coscas G. Age-related macular degeneration. Presse Méd 2002; Aug 24 31(27):1282-7.

05. Ferris FL. Senile macular degeneration: review of epidemiologic features. Am J Ophthalmol 1983;118(2):132-51.

06. Abujamra S, Ávila M, Barsante C, Farah ME, Gonçalves JOR, Lavinsky J at al. Retina e Vítreo- clínica e cirurgia. São Paulo: Rocca; 2000.

07. Bressler NM, Bressler SB, Fine SL. Age- related macular degeneration. Surv Ophthalmol 1988; 32(6):375-413.

08. Oliveira LRM. Classificação dos deficientes visuais e sua reabilitação. In: IV Congresso Brasileiro de Prevenção da Cegueira; Belo Horizonte: 1980; 2:439-44.

09. Organização Mundial de Saúde. 0 atendimento de crianças com baixa visão. Programa para prevenção de cegueira da OMS. Bangok: OMS 1994. p.32-9.
10. Castro DDM. Visão subnormal. Rio de Janeiro: Cultura Médica;1994. 168p.

11. Knave B, Tengroth B, Voss M. Age and sex distribution of some retinal macular diseases: senile and presenile macular degeneration and central serous retinitis. Acta Ophthalmol Suppl 1984;161:95-103.

12. Owen CG, Fletcher AE, Donoghue M, Rudnicka AR. How big is the burden of visual loss caused by age related macular degeneration in the United Kingdom ? Br J Ophthalmol 2003; 87(3):312-7.

13. Angelucci RI, Tayah D, Serra DC, Sampaio PR, Rehder JRCL. Serviço de visão subnormal da disciplina de Oftalmologia da FMABC- Análise dos cem primeiros casos com diagnóstico de visão subnormal. Arq Méd ABC 2003;28(1):7-9.

14. Portal da Retina. Carvalho KMM. Reabilitação visual em DMRI. Disponível em URL: http://www.portaldaretina.com.br/home/artigos.asp?id=22 Acessado em 01 de junho de 2004.

15. Buchignani BPC, Silva, MRBM. Serviço de visão subnormal do Hospital das Clínicas de Botucatu: levantamento das causas e resultados. Rev bras Oftal 1991;50(5):305-10.

16. Tayah D. Custo benefício da implantação do serviço de visão subnormal no Instituto de Olhos do ABC. Tese (Mestrado). Universidade Federal de São Paulo - EPM. São Paulo, 2002. 